\title{
MOTIVACIONES \\ QUE IMPULSAN AL CAMBIO \\ DE TRAYECTORIA VITAL \\ EN LOS EMPRENDEDORES

\author{
(PASTO, COLOMBIA, \\ 2012-2013)
}

\section{RESUMEN}

Este documento es producto de una investigación, cuyo objetivo general fue analizar las motivaciones que impulsan al cambio de trayectoria vital en los emprendedores de la ciudad de Pasto. Se trabajó con creadores de microempresas y pequeñas empresas, con quienes, bajo el enfoque de investigación cuantitativo, método empírico-analítico y tipo descriptivo, a través de una encuesta, se identificaron dichas motivaciones y se determinó cuáles de ellas tuvieron mayor incidencia. Para un mejor manejo de la información, se clasificaron las motivaciones de emprendimiento según la adaptación realizada por Rodrigo Varela sobre la teoría de Albert Shapero en cuanto a la clasificación de impulsos positivos, neutros y negativos, en la cual se tienen en cuenta componentes socioculturales, educativos e investigativos que constituyen niveles de apreciación para desentrañar motivaciones particulares que hacen posible una acción empresarial, a partir de conocimientos, valores, aptitudes, actitudes y competencias.

Palabras clave: Emprendedor; Emprendimiento; Empresa; Motivaciones de emprendimiento. 


\section{CLAUDIA MAGALI SOLARTE SOLARTE* \\ MARTHA LIDA SOLARTE SOLARTE * \\ CARLOS ARCOS GUERRERO *}

\section{MOTIVATIONS THAT DRIVE THE CHANGE OF LIFE COURSE IN ENTREPRENEURSHIP (PASTO, COLOMBIA, 2012-2013)}

\section{ABSTRACT}

The paper is the result of an investigation; the overall objective was to analyze the motivations that drive the change of life course entrepreneurs in the city of Pasto. We worked with creators of micro and small enterprises, with whom, under the approach of quantitative research, empirical-analytical method and descriptive; through a survey, these motivations are identified and determined which ones had the highest incidence. For better information management, entrepreneurial motivations were classified as adapted by Rodrigo Varela on the theory of Albert Shapero regarding the classification of positive, neutral and negative pulses, where components are taken into account socio-cultural, educational and research constituting levels of appreciation to unravel particular motivations that make a corporate action, that takes into account knowledge, values, skills, attitudes and competences.

Keywords: Entrepreneurship; Entrepreneurial; Enterprises; Entrepreneurial Motivations.

\section{INTRODUCCIÓN}

El ser humano desarrolla su existencia en interacción con su medio, pero no solamente de manera contemplativa, sino transformadora. Por consiguiente, es importante estudiar las motivaciones que las personas tienen para crear empresas de diversa índole, lo cual permitirá hacer un análisis sobre cómo estas motivaciones son el resultado, no solo de la espontaneidad o impulsos ante coyunturas sociales o económicas, sino que existen antecedentes que están en relación con motivaciones personales o familiares, niveles de educación y condiciones de contexto.

Si bien la creación de empresas es un proceso que se puede dar en cualquier lugar, es también cierto que cada una de ellas es el resultado de la acción de las personas (Shapero, citado en Varela, 2008), y estas son producto del contexto y de la acción educativa; es importante identificar y evaluar ideas empresariales, las cuales pueden reflejar una idiosincrasia y un tipo de educación, bajo el cual se pueda tener en cuenta o no la formación de emprendedores y, para el caso de los programas de Administración de empresas, cómo estos fortalecen el espíritu empresarial y su accionar en emprendimientos reales, y su incidencia en el sector productivo local, regional y nacional.

\footnotetext{
* Magíster, docente investigadora, Institución Universitaria CESMAG. Colombia. Correo-e: cmsolarte@iucesmag.edu.co.

* Especialista, docente investigadora, Institución Universitaria CESMAG. Colombia. Correo-e: mlsolarte@iucesmag.edu.co.

* Magíster, investigador, Institución Universitaria cesmag. Colombia. Correoe: saudade.ca@gmail.com.

Recibido: 20 de noviembre de 2014, aceptado: 12 de marzo de 2015.

Para citar el artículo: Solarte, C.M.; Solarte, M.L. y Arcos, C. (2015). "Motivaciones que impulsan al cambio de trayectoria vital en los emprendedores (Pasto, Colombia, 2012-2013)", en Sotavento MBA, n. ${ }^{\circ} 25$, pp. 48-55. D0I: http://dx.doi. org/10.18601/ $01233734 . n 25.06$
} 


\section{FUNDAMENTOTEÓRICO}

La investigación acerca del fenómeno emprendedor continúa fortaleciéndose por los beneficios que genera para la sociedad y crea una cultura a través de las prácticas empresariales, lo cual implica el desarrollo humano y social, constituyéndose en agentes de cambio orientados al mejorestar de las familias con la generación de empleo.

Dentro de los conceptos y enfoques sobre emprendimiento, se puede considerar el pensamiento anglosajón y europeo, y el de algunos latinoamericanos.

Algunos conceptos resultan técnicos, como la definición que da Halloran: "El emprendedor es aquel que asume el riesgo de obtener beneficios o incurrir en pérdidas... es un empresario potencial que lleva a cabo una aventura, la organiza, busca capital para financiarla y asume el riesgo" (citado en Castillo, 2006, pág. 39), donde se puede apreciar que se delega la responsabilidad del emprendimiento a individuos o comunidades, bajo una visión "aventurera" de quien trata de conquistar, en los ámbitos socioeconómicos, un puesto que quizá no le corresponda.

Pensamiento diferente al de Ovalle (2012, párr. 6), quien lo considera como agente movilizador de la economía, inserto en un ecosistema emprendedor donde todos generan acciones de reciprocidad hacia los nuevos empresarios. Existe una correlación de fuerzas sociales en las que, si bien el emprendedor puede ser protagonista, también hace parte de un sistema solidario de prácticas que contribuyen al logro de determinado objeto.

Por otro lado, están las concepciones globalizantes y neutras a los contextos, donde el emprendedor es el mismo en cualquier lugar del mundo: "creo que el perfil psicográfico del emprendedor es el mismo, se trate de un chileno, noruego o japonés" (Thiers, 2002, párr. 9). Si bien se puede aceptar que los emprendedores tienen una serie de características comunes, sus motivaciones son variadas, lo cual los convierte en innovadores de ideas existentes, y su mapa mental es enriquecido de acuerdo con su concepción de la vida.

En cuanto a motivaciones, se hace referencia a elementos con causa que interactúan para formar un producto o provocar una situación determinada, así como motivaciones de emprendimiento como factores que impulsan a las personas a convertirse en empresarias bajo componentes socioculturales, educativos e investigativos que generan el cambio de trayectoria vital. En ese sentido, "Una motivación es una tendencia persistente, una inclinación permanente a realizar cierta clase de actos y experimentar cierta clase de sentimientos en cierta clase de situaciones" (Geertz, 1997, pág. 93).

McClelland (1961, 1989), en sus investigaciones sobre las motivaciones humanas y las relaciones de estas con el crecimiento económico mediante el desarrollo del espíritu empresarial, lo relaciona con el deseo de logro, es decir, hacer las cosas mejor y que le generen satisfacción no solamente económica, sino por el desarrollo de capacidades, poder o liderazgo.

Por su parte, Jeffrey Timmons (2004), respecto a motivaciones hacia el liderazgo y espíritu empresarial, destaca cualidades como la imaginación, compromiso, pasión, integridad, trabajo de equipo y visión.

Petri y Govern (2006) relacionan la motivación con la conducta y la forma como esta actúa. Explican por qué la conducta se realiza en una situación y no en otra. Entre tanto, Vroom, según su teoría de expectativas, observa la motivación como un proceso de elección entre varios estímulos: se da un determinado 
comportamiento porque se esperan ciertos resultados (citado en Dalton, M., Hoyle, D. \& Watts, M., 2007).

Dalton, Hoyle \& Watts (2007) consideran la motivación desde la psicología, sociología y antropología, de la siguiente manera: desde la primera, la motivación se centra en la conducta de los individuos y en los factores que los impulsan a comportarse en una forma determinada. La sociología se centra en la interacción de dos o más individuos y en sus relaciones en situaciones colectivas; según esta, los individuos funcionan en comunidades. Por su parte, la antropología se centra en la cultura, y cómo ella se fortalece a través de diferentes motivaciones que los individuos tienen, entre las que pueden estar el trabajo y la economía.

El profesor Varela (2008) expresa que la primera etapa para la creación de empresas es la motivacional o de gusto, que tiene que ver con factores educativos, culturales, sociales y del ambiente.

En cuanto al estudio de motivaciones empresariales, se han planteado diferentes modelos como la Teoría de la Acción planeada, de Ajzen, quien tiene en cuenta tres elementos: la actitud hacia la conducta, creencias normativas y de control conductual. Este se basa en la Teoría del Evento empresarial, de Shapero y Sokol, según la cual "la idea de creación de empresas y de nuevos empresarios, surge a partir de la interacción de factores sociales, psicológicos, culturales y económicos, los cuales se integran en la percepción de deseabilidad y la percepción de factibilidad" (Shapero \& Sokol, citados por Cabrera, 2009, pág. 34). Está, también, el Modelo de Intenciones empresariales o actitudes, de Liñan y Rodríguez, en el cual se plantea la predisposición del sujeto para llevar a cabo una idea de negocio y transformarla en una empresa según su experiencia, creencias, educación y modelos sociales ( Liñan \& Rodríguez, citados en Cabrera, 2009).

\section{METODOLOGÍA}

El estudio se realizó bajo el enfoque cuantitativo de investigación. Se recopiló información sobre las motivaciones que impulsan al emprendimiento y su grado de incidencia en la creación de empresas, para describir el objeto de estudio a través de la medición de las variables de interés. La población estuvo conformada por gestores de empresa, según información de la Cámara de Comercio de Pasto. Se trabajó con una muestra probabilística conformada por 347 emprendedores en los siguientes sectores: comercio; industria manufacturera; actividades de servicios, construcción, alojamiento y servicios de comida; agricultura y pesca; información y comunicaciones, y actividades profesionales $\mathrm{y}$ técnicas; transporte y almacenamiento; educación; actividades inmobiliarias y de alquiler; actividades de atención en salud humana y asistencia social; explotación de minas, y actividades financieras y de seguros.

Para la recolección de información se elaboró un cuestionario que permitió identificar las motivaciones de emprendimiento. Este constó de 39 preguntas divididas en siete apartes que proporcionaron información demográfica, socioeconómica, emprendimiento, sobre la empresa creada, motivaciones de emprendimiento y gestión de recursos.

Los resultados del instrumento fueron organizados y sistematizados en programa Excel. El análisis se orientó a los mayores y menores porcentajes encontrados en cada una de las preguntas y la relación entre variables e indicadores. Para el manejo de la información se acogió la adaptación que realizó Rodrigo Varela, con base en la teoría de Albert Shapero, sobre "Fuerzas para el cambio de trayectoria vital", el cual considera tres tipos de motivaciones: positivas, negativas y neutras; a estas se agregaron otras motivaciones, que por su "idealismo" se las denominó motivaciones altruistas. 


\section{RESULTADOS}

\section{CARACTERIZACIÓN DEL EMPRENDEDOR}

Los encuestados fueron del género masculino, $64 \%$ y del género femenino, $36 \%$. Con edades entre 31 y 40 años el $32 \%$; entre 41 a 50 años, el $26 \%$; entre 21 y 30 años, el $21 \%$; entre 51 y 60 años, el $11 \%$; más de 61 años, el $8 \%$, y el $2 \%$, con menos de 20 años. En cuanto a estudios realizados, el $34 \%$ pertenece al nivel profesional; el $21 \%$, a estudios de posgrado; el $18 \%$, bachiller académico y normalista; el $11 \%$, tecnología; el $9 \%$, técnico; el $3 \%$, primaria; el $2 \%$, ningún estudio, y el $1 \%$, maestría. En el nivel profesional, el $63 \%$ pertenece a ciencias económicas y administrativas; el $14 \%$, a ingenierías; el $6 \%$, a artes; el $5 \%$, a ciencias de la salud y derecho, respectivamente; el $2 \%$, a ciencias de la educación y ciencias humanas, y el $1 \%$, a ciencias agrícolas, ciencias exactas y naturales y ciencias pecuarias.

Para la creación de la empresa, los emprendedores realizaron estudios preliminares como: estudio de mercado, $21 \%$; no realizó ningún estudio, $17 \%$; estudio financiero, $16 \%$; de factibilidad, $12 \%$, y el $10 \%$ realizó estudios técnicos y administrativos, respectivamente; el $8 \%$, de impacto socioeconómico; el $4 \%$, de impacto ambiental, y el $2 \%$, otros. En cuanto a capacitación antes de crear la empresa, el $16 \%$, en administración; el $13 \%$ no realizó ninguna; el $12 \%$, en mercadeo; el $11 \%$, en formulación de proyectos; el $11 \%$, otros; el $9 \%$, en contabilidad; el $7 \%$, en manejo de talento humano y publicidad, y el $5 \%$ restante, en gestión de recursos. Al $57 \%$ el estudio realizado sí lo ha guiado en el desarrollo de su empresa, en tanto al $16 \%$ no, y el $27 \%$ no dio respuesta.

\section{IDENTIFICACIÓN DE MOTIVACIONES}

En el estudio realizado en la ciudad de Pasto ( Nariño-Colombia), en 2012-2013, se identificaron 75 motivaciones que han impulsado al cambio de trayectoria vital en los emprendedores. Dentro de estas, aquellas de mayor incidencia se ubicaron en porcentajes como positivas, neutras, negativas y altruistas (véase Figura 1 ).

Figura 1. Clasificación de las motivaciones

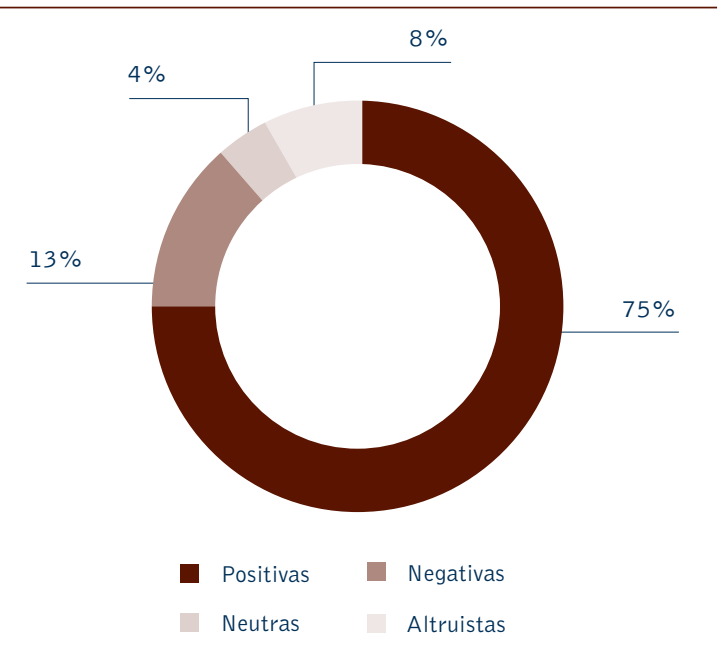

Fuente: Elaboración propia.

A la pregunta: De las motivaciones señaladas anteriormente ¿Cuál considera usted que fue la de mayor incidencia para crear su empresa?, los encuestados respondieron: en las motivaciones positivas: la capacidad de gestión, el conocimiento del negocio y el ser visionario (véase Figura 2); en las motivaciones neutras: tener un cambio de vida laboral y terminación de un ciclo formativo (véase Figura 3); en las negativas: Ia falta de proyección (véase Figura 4), y dentro de las motivaciones altruistas: lograr independencia y cambiar de empleado a empresario (véase Figura 5). 
Figura 2. Motivaciones de mayor incidencia para crear empresa -positivas-

\begin{tabular}{|c|c|c|}
\hline Espíritu emprendedor & $1 \%$ & \\
\hline Familiares empresarios & $1 \%$ & \\
\hline Disponibilidad de financiamiento & $1 \%$ & \\
\hline Talento empresarial y administrativo & $1 \%$ & \\
\hline Habilidades empresariales & $1 \%$ & \\
\hline Es visionario & & $2 \%$ \\
\hline Conocimientos de negocios & & $2 \%$ \\
\hline Capacidad de gestión & & $2 \%$ \\
\hline
\end{tabular}

Fuente: Elaboración propia.

Figura 3. Motivaciones de mayor incidencia para crear empresa -neutras-

Tener un cambio de vida laboral $\overline{50 \%} 5$

Fuente: Elaboración propia.

Figura 4. Motivación de mayor incidencia para crear empresa -negativas-

\begin{tabular}{rll}
\hline Ingresos insuficientes & $20 \%$ \\
Aburrimiento en la actividad que estaba realizando & $20 \%$ \\
Despido o retiro de la actividad actual & $20 \%$ \\
\hline
\end{tabular}

Fuente: Elaboración propia.

\section{CONCLUSIONES Y DISCUSIÓN}

El mayor porcentaje de emprendedores se encontró en edades entre 31 y 40 años, gente adulta, representantes de dos generaciones, con quienes la creación de empresa se da más por el pragmatismo; "la capacidad de realización, es entendida como el pragmatismo necesario para poner en ejecución las ideas y proyectos que se hayan generado" (Varela, 2008, pág. 19), como una de las 24 características nombradas por el autor que colaboran en el desarrollo del espíritu empresarial.

En cuanto a las motivaciones clasificadas como positivas, como la más relevante aparece el conocimiento del negocio; esto puede tener diferentes fuentes, algunas de las cuales pueden ser la experiencia en el negocio, estudios realizados, capacitaciones por parte de entidades especializadas y el aporte de las nuevas tecnologías de la información y la comunicación. 
Figura 5. Motivación de mayor incidencia para crear empresa -altruistas-

\begin{tabular}{|c|c|c|}
\hline Por vocación & $3 \%$ & \\
\hline Ofrecer un mejor servicio & $3 \%$ & \\
\hline Deseo de que la empresa continue & $3 \%$ & \\
\hline Colaboración a la familia & $3 \%$ & \\
\hline Convicción en una religión & $3 \%$ & \\
\hline Poner ideas en marcha & $3 \%$ & \\
\hline Ser empresario & $3 \%$ & \\
\hline Capacidad para asumir retos & $6,1 \%$ & \\
\hline Confianza en sí mismo y capacidades & $6,1 \%$ & \\
\hline Superación personal & $6,1 \%$ & \\
\hline Contribuir al desarrollo regional & $12,1 \%$ & \\
\hline Cambiar de empleado a empresario & $18,2 \%$ & \\
\hline Lograr independencia & & $30,3 \%$ \\
\hline
\end{tabular}

Fuente: Elaboración propia.

Para las motivaciones neutras más relevantes: el tener un cambio de vida laboral y la terminación de un ciclo formativo fue fundamental para crear empresa.

Para el caso de las negativas, aparece la falta de proyección social y económica, es decir, con el emprendimiento se trata de solventar esta carencia en un territorio considerado de bajo desarrollo. Pasto es una ciudad intermedia por su tamaño y número de habitantes, comparada con las capitales de departamento consideradas como polos de desarrollo de Colombia. Hace parte de los territorios de periferia, considerados al margen de proyectos de desarrollo nacional, lo cual puede motivar a emprender.

Las motivaciones altruistas que se citan como de mayor incidencia, son: lograr independencia y el cambio de empleado a empresario. Las dos están relacionadas y constituyen un anhelo generalizado en una región y en un país donde las condiciones de los trabajadores dependientes no son las mejores, y la mayoría de los encuestados tenían una condición de empleados, lo cual es verdad en cuanto a que se ha inculcado una creencia, errónea en el sentido de continuar con la falacia de la independencia económica, confundiéndola, quizá, con la independencia política; pero, para el caso de la economía y, particularmente, de una empresa, en su proceso productivo, inversiones, organización, decisiones y otros, se encuentra siempre en interdependencia con otros factores externos.

Si bien las motivaciones manifestadas hacen parte de inquietudes emprendedoras y de un espíritu empresarial implícito, también indican dispersión en las ideas y la ausencia de una cultura del emprendimiento, la cual encausaría estas inquietudes hacia mejores resultados; pero, sin delegar toda la responsabilidad en los individuos o en las comunidades, sino en un trabajo mancomunado con las instituciones, la educación, las políticas públicas y un manejo adecuado de la economía en general. 
La clasificación de motivaciones positivas, negativas, neutras y altruistas no obedece a algo instrumental, sino que en ella se tienen en cuenta componentes socioculturales, educativos e investigativos que generan el cambio de trayectoria vital, y que en el presente estudio constituyeron niveles de apreciación necesarios para desentrañar motivaciones particulares que hacen posible una acción empresarial, en la que se tienen en cuenta conocimientos, valores, aptitudes, actitudes y competencias.

\section{REFERENCIAS}

Cabrera, N. (2009). Actitudes hacia la creación de empresas en estudiantes de Ingeniería agroindustrial y Administración de empresas de la Universidad de Nariño (Trabajo de grado). Pasto: Universidad de Nariño.

Castillo, D. (2006). Impacto generado en el desarrollo regional por la gestión de la unidad de emprendimiento del Centro multisectorial Lope del Municipio de Pasto, SENa regional Nariño (Trabajo de grado). Pasto: Universidad de Nariño.

Dalton, M.; Hoyle, D. \& Watts, M. (2007). Relaciones humanas. México: Thomson.

Geertz, C. (1997). La interpretación de las culturas. Barcelona: Gedisa S.A.

Gobernación de Nariño (2012). Plan de desarrollo Departamento de Nariño 2012-2015 "Nariño mejor". Disponible en http://www.narino. gov.co/index.php/plan-de-desarrollo-2012-2015. Consultado el 19 de febrero de 2013.

McCleland, D. (1961). The Achieving Society. New York: McMillan.

McClelland, D. (1989). Estudio de la motivación humana. Madrid: Narcea.

Ministerio de Comercio, Industria y Turismo (2009, 3 de abril). Decreto 1192. Por el cual se reglamenta la Ley 1014 de 2006, sobre el fomento a la cultura del emprendimiento y se dictan otras disposiciones. Disponible en http://actualicese.com/normatividad/2009/04/03/decreto-1192-de-03-04-2009/. Consultado el 10 noviembre de 2012.

Ovalle, P. (2012). El emprendimiento en Chile. Universidad de Chile -Diario electrónico. Disponible en http://radio.uchile.cl/opiniones/139850/ comment-page-1/\#comment-66888. Consultado el 30 de septiembre de 2013.

Petri, H. \& Govern, J. (2006). Motivación: teoría, investigación y aplicaciones. México:Thomson

Thiers, E. (2002). El emprendimiento en América Latina. Disponible en http://www.ceo. cl/609/article-16187.html. Consultado el 20 de junio de 2012.

Timmons, J. (2004). New venture creation. Entrepreneurship for the $21^{\text {st }}$ century. Boston: McGraw Hill.

Varela, R. (2008). Innovación Empresarial. Arte y Ciencia en la Creación de Empresas. 3. ${ }^{a}$ ed. Bogotá: Pearson Prentice Hall.

Vélaz, J. (1996). Motivos y motivación en la empresa. Madrid: Díaz de Santos S.A. 